Article

\title{
Evaluation of Four Different Analytical Tools to Determine the Regional Origin of Gastrodia elata and Rehmannia glutinosa on the Basis of Metabolomics Study
}

\author{
Dong-Kyu Lee ${ }^{1}$, Dong Kyu Lim ${ }^{1}$, Jung A. Um ${ }^{1}$, Chang Ju Lim ${ }^{1}$, Ji Yeon Hong ${ }^{1}$, \\ Young A Yoon ${ }^{2}$, Yeonsuk Ryu ${ }^{2}$, Hyo Jin Kim ${ }^{3}$, Hi Jae Cho ${ }^{4}$, Jeong Hill Park ${ }^{1}$, \\ Young Bae Seo ${ }^{5}$, Kyunga Kim ${ }^{6}$, Johan Lim ${ }^{7}$, Sung Won Kwon ${ }^{1, *}$ and Jeongmi Lee ${ }^{2, *}$ \\ 1 College of Pharmacy, Seoul National University, Seoul 151-742, Korea; \\ E-Mails: dongqchicken@snu.ac.kr (D.-K.L.); ddongq1989@snu.ac.kr (D.K.L.); \\ jafirst11@snu.ac.kr (J.A.U.); limcj85@snu.ac.kr (C.J.L.); vastgy88@snu.ac.kr(J.Y.H.); \\ hillpark@snu.ac.kr (J.H.P.) \\ 2 School of Pharmacy, Sungkyunkwan University, Suwon 440-746, Korea; \\ E-Mails: duddk1987@nate.com (Y.A.Y.); ryu.yeonsuk@gmail.com (Y.R.) \\ 3 College of Pharmacy, Dongduk Women's University, Seoul 136-714, Korea; \\ E-Mail: hyojkim@dongduk.ac.kr
}

4 Korea Promotion Institute for Traditional Medicine Industry, Gyeongsan 712-260, Korea; E-Mail: choze@naver.com

5 Department of Herbology, College of Oriental Medicine, Daejeon University, Daejeon 300-716, Korea; E-Mail: genin@dju.kr

6 Department of Statistics, Sookmyung Women's University, Seoul 140-742, Korea; E-Mail: kyunga@sookmyung.ac.kr

7 Department of Statistics, Seoul National University, Seoul 151-742, Korea; E-Mail: johanlim@snu.ac.kr

* Authors to whom correspondence should be addressed; E-Mails: swkwon@snu.ac.kr (S.W.K.); jlee0610@skku.edu (J.L.); Tel.: +82-2-880-7844 (S.W.K.); +82-31-290-7784 (J.L.); Fax: +82-31-876-8800 (S.W.K.); +82-31-292-8800 (J.L.).

Received: 11 March 2014; in revised form: 2 May 2014 / Accepted: 13 May 2014 / Published: 16 May 2014

\begin{abstract}
Chemical profiles of medicinal plants could be dissimilar depending on the cultivation environments, which may influence their therapeutic efficacy. Accordingly, the regional origin of the medicinal plants should be authenticated for correct evaluation of their medicinal and market values. Metabolomics has been found very useful for
\end{abstract}


discriminating the origin of many plants. Choosing the adequate analytical tool can be an essential procedure because different chemical profiles with different detection ranges will be produced according to the choice. In this study, four analytical tools, Fourier transform near-infrared spectroscopy (FT-NIR), ${ }^{1} \mathrm{H}$-nuclear magnetic resonance spectroscopy ( $\left.{ }^{1} \mathrm{H}-\mathrm{NMR}\right)$, liquid chromatography-mass spectrometry (LC-MS), and gas chromatography-mass spectroscopy (GC-MS) were applied in parallel to the same samples of two popular medicinal plants (Gastrodia elata and Rehmannia glutinosa) cultivated either in Korea or China. The classification abilities of four discriminant models for each plant were evaluated based on the misclassification rate and $\mathrm{Q}^{2}$ obtained from principal component analysis (PCA) and orthogonal projection to latent structures-discriminant analysis (OPLS-DA), respectively. ${ }^{1} \mathrm{H}-\mathrm{NMR}$ and LC-MS, which were the best techniques for $G$. elata and $R$. glutinosa, respectively, were generally preferable for origin discrimination over the others. Reasoned by integrating all the results, ${ }^{1} \mathrm{H}-\mathrm{NMR}$ is the most prominent technique for discriminating the origins of two plants. Nonetheless, this study suggests that preliminary screening is essential to determine the most suitable analytical tool and statistical method, which will ensure the dependability of metabolomics-based discrimination.

Keywords: traditional medicine; Gastrodia elata; Rehmannia glutinosa; plant metabolomics; origin discrimination; multivariate statistical analysis

\section{Introduction}

Herbal medicines have been traditionally used to treat patients [1]. They contain a variety of natural pharmacologically-active compounds, which often have synergistic or complex remedial effects with fewer side effects than synthetic drugs [2-4]. Medicinal plants, which are the major source of Traditional Chinese Medicines (TCMs), are mainly produced in Asian countries, including Korea and China. Though medicinal plants of identical species may be used for the same healing purposes, their chemical profiles could differ because of differences in their cultivation environments. A great deal of literature has reported the effects of climate, pesticides, fertilizer, and abiotic stress on plant metabolism [5-8]. Changes in the chemical composition of plants may influence their therapeutic efficacy, which in turn may affect their quality. Therefore, the regional origin of medicinal plants should be confirmed to evaluate their medicinal and market value.

Currently, medicinal plant species and origins are authenticated largely by evaluating their phenotype. Though this method is simple, rapid, and requires only an expert's knowledge and experience, it lacks the objectivity of scientific testing, and, therefore, it is not appropriate for quality control of medicinal plants. Moreover, phenotype evaluation is practically impossible when morphological features of the plants are destroyed by processing, such as grinding [9]. Metabolomic approaches can overcome these limitations and have been suggested to detect metabolic differences arising from genetic or environmental effects.

Metabolomic profiles have been obtained by various analytical techniques including fourier transform near-infrared (FT-NIR) spectroscopy [10], ${ }^{1} \mathrm{H}$-nuclear magnetic resonance $\left({ }^{1} \mathrm{H}-\mathrm{NMR}\right)$ spectroscopy [11], 
liquid chromatography-mass spectrometry (LC-MS) [12], and gas chromatography-mass spectroscopy (GC-MS) [13]. FT-NIR allows for the rapid and non-destructive analysis of the metabolome, though quantitative data at the compound level is difficult to obtain [14]. NMR ensures reproducible and rapid analysis; however, its low sensitivity prevents quantifying metabolites at low concentrations [15]. MS can be advantageous over ${ }^{1} \mathrm{H}-\mathrm{NMR}$ and FT-NIR given that it provides higher sensitivity and broader detection range despite its relatively poor reproducibility [16]. Accordingly, researchers need to select a suitable approach depending on their objective. For metabolomics-based authentication of medicinal plant origins, choosing the adequate analytical tool is essential because the choice will affect the chemical profile and detection range [17]. In this study, four analytical tools, FT-NIR, LC-MS,

GC-MS, and ${ }^{1} \mathrm{H}-\mathrm{NMR}$, were used in parallel to determine the regional origin (Korea vs. China) of two traditional medicinal plants, Gastrodia elata and Rehmannia glutinosa, which are commonly used in Asian countries. G. elata is known to possess hypoglycemic [18], hemostatic and immunologic [19] activities and $R$. glutinosa is known for its anti-convulsive [20], anti-inflammatory and anti-angiogenic effects [21]. Using eight discriminant models derived from the metabolic profiling and two multivariate statistical analyses, we have attempted to determine the best approach for each species.

\section{Results and Discussion}

\subsection{Metabolomic Profiling and Putative Identification of Metabolites with Four Analytical Approaches}

Experimental conditions including sample preparation and instrumental setting were optimized for each analytical approach based on literatures and our own experiments. As a result, metabolomic profiles were successfully obtained for all tested samples using four analytical approaches: FT-NIR (Supplementary Figure S1), ${ }^{1}$ H-NMR (Supplementary Figure S2), LC-MS (Supplementary Figure S3), and GC-MS (Supplementary Figure S4). As reviewed previously [14,16,17], each analytical approach had advantages and disadvantages. In the FT-NIR analysis, which has been suggested as a first-round screening technique for metabolic fingerprinting [22], sample preparation was convenient, but identifying spectral peaks arising from complex functional groups was extremely difficult. Relatively, it is possible for LC-MS and GC-MS to separate and identify the peaks of a large number of metabolites.

For the LC-MS analysis, inconsistent retention times and fragment patterns in the spectra have prevented the development of a public database; therefore, the use of standard compounds was necessary to identify metabolites. In addition, assigning secondary metabolites requires separating compounds at baseline, which can be time-consuming. Although a number of secondary metabolites in Schisandra chinensis were successfully assigned in our previous study [23] by using the literature on the elution order and mass spectra, the identification process was not simple and was possible only because there was enough literature on the secondary metabolites in S. chinensis. For ${ }^{1} \mathrm{H}-\mathrm{NMR}$ and GC-MS, the databases from BMRB and NIST, respectively, made putative peak assignments convenient. As summarized in Table 1, nine metabolites were assigned in each plant by ${ }^{1} \mathrm{H}-\mathrm{NMR}$ analysis in this study. From the GC-MS analysis, 35 metabolites from G. elata (Table 2) and 29 from $R$. glutinosa (Table 3) were putatively assigned in their derivatized forms. 
Table 1. Representative chemical shifts of metabolites putatively identified in G. elata and R. glutinosa using ${ }^{1} \mathrm{H}-\mathrm{NMR}$ spectroscopy.

\begin{tabular}{cccc}
\hline \multicolumn{2}{c}{ G. elata } & \multicolumn{2}{c}{ R. glutinosa } \\
\hline Metabolites & Chemical shift (ppm) & Metabolites & Chemical shift (ppm) \\
\hline phenylalanine $^{b}$ & 7.38 & catalpol $^{a}$ & $6.47,5.04$ \\
gastrodine $^{a}$ & $7.33,7.04$ & sucrose $^{b}$ & 5.40 \\
4-hydroxybenzyl alcohol $^{a}$ & $7.24,6.84$ & glucose $^{b}$ & 5.23 \\
sucrose $^{b}$ & 5.40 & glycine $^{b}$ & 3.62 \\
glutamate $^{b}$ & $2.40,2.05$ & arginine $^{b}$ & $3.23,1.71$ \\
acetate $^{b}$ & 1.93 & $\gamma$-aminobutyric acid $^{b}$ & 3.00 \\
alanine $^{b}$ & 1.48 & acetate $^{b}$ & 1.93 \\
threonine $^{b}$ & 1.33 & alanine $^{b}$ & 1.48 \\
valine $^{b}$ & 1.00 & threonine $^{b}$ & 1.33 \\
\hline
\end{tabular}

${ }^{a}$ assigned by data from literatures; ${ }^{b}$ identified by BMRB database.

Table 2. Retention times, mass fragments, and match factors of detected metabolites in G. elata by GC-MS.

\begin{tabular}{|c|c|c|c|c|}
\hline No. & $\mathbf{t}_{\mathrm{R}}(\mathrm{min})$ & Identified metabolites & Mass fragments (m/z) & Match factor $^{a}$ \\
\hline 1 & 6.72 & alanine & $59,73,116,147,190$ & 89 \\
\hline 2 & 7.08 & glycine & $52,73,102,147$ & 91 \\
\hline 3 & 8.17 & disiloxane & $73,103,131,147,227$ & 83 \\
\hline 4 & 9.50 & valine & $52,73,117,144,218$ & 80 \\
\hline 6 & 11.13 & glycerol & $73,103,117,147,205,218$ & 89 \\
\hline 7 & 11.82 & glycine & $73,100,147,174,248$ & 90 \\
\hline 8 & 13.37 & serine & $73,100,147,204,218$ & 88 \\
\hline 9 & 14.05 & threonine & $73,101,117,147,218$ & 84 \\
\hline 10 & 16.63 & malic acid & $55,73,133,147,189,233$ & 83 \\
\hline 11 & 16.75 & silane & $73,147,179,253,268$ & 88 \\
\hline 12 & 17.17 & threitol & $73,103,117,147,217$ & 91 \\
\hline 13 & 17.33 & proline & $73,156,230,258$ & 91 \\
\hline 14 & 17.38 & 3,8-dioxa-2,9-disiladecane & $73,103,147,205,217$ & 89 \\
\hline 15 & 17.52 & aspartic acid & $73,100,147,218,232$ & 82 \\
\hline 16 & 19.17 & 2,3,4-trihydroxybutyric acid & $73,147,204,220,292$ & 86 \\
\hline 17 & 21.62 & $\begin{array}{l}\text { 2,3,4,5-tetrahydroxypentanoic } \\
\text { acid-1,4-lactone }\end{array}$ & $73,102,117,147,189,217$ & 82 \\
\hline 18 & 23.28 & D-ribose & $73,103,147,217,307$ & 84 \\
\hline 19 & 23.40 & D-xylose & $73,103,147,217,307$ & 84 \\
\hline 20 & 25.30 & arabinitol & $73,103,147,217$ & 81 \\
\hline 21 & 26.57 & D-glucitol & $73,103,117,147,217$ & 84 \\
\hline 22 & 28.00 & 1,2,3-propanetricarboxylic acid & $73,147,273$ & 91 \\
\hline 23 & 28.10 & pentaric acid & $73,147,245,273,319$ & 83 \\
\hline 24 & 30.63 & galactose oxime & $73,103,147,205,319$ & 84 \\
\hline 25 & 30.91 & glucitol & $73,147,205,319$ & 81 \\
\hline 26 & 31.17 & glucose oxime & $73,103,147,217$ & 84 \\
\hline 27 & 31.22 & ribitol & $73,103,147,217$ & 87 \\
\hline
\end{tabular}


Table 2. Cont.

\begin{tabular}{lllll}
\hline No. & $\mathbf{t}_{\mathbf{R}}(\mathbf{m i n})$ & Identified metabolites & Mass fragments $(\mathbf{m} / \mathbf{z})$ & Match factor $^{a}$ \\
\hline 28 & 32.63 & hexadecanoic acid & $55,73,117,145,313$ & 87 \\
29 & 33.05 & myo-inositol & $73,147,191,217,318$ & 82 \\
30 & 34.22 & myo-inositol & $73,147,217,305$ & 87 \\
31 & 35.87 & 9,12 -octadecadienoic acid & $75,95,117,129,337$ & 92 \\
32 & 36.45 & octadecanoic acid & $55,73,117,145,341$ & 90 \\
33 & 42.37 & hexadecanoic acid & $57,73,147,239,371$ & 82 \\
34 & 43.92 & D-glucopyranoside & $73,103,147,217,361$ & 91 \\
35 & 45.13 & monostearin & $57,73,147,399$ & 89 \\
\hline
\end{tabular}

${ }^{a}$ full score at 100.

Table 3. Retention times, mass fragments, and match factors of detected metabolites in R. glutinosa by GC-MS.

\begin{tabular}{|c|c|c|c|c|}
\hline No. & $\mathbf{t}_{\mathrm{R}}(\mathrm{min})$ & Identified metabolites & Mass fragments $(\mathrm{m} / \mathrm{z})$ & Match factor $^{a}$ \\
\hline 1 & 7.92 & propanoic aicd & $73,117,147,191$ & 90 \\
\hline 2 & 9.06 & alanine & $73,116,147$ & 83 \\
\hline 3 & 9.95 & ethanedioic acid & $73,147,196$ & 90 \\
\hline 4 & 12.24 & L-valine & $73,144,218$ & 80 \\
\hline 5 & 13.80 & L-leucine & $73,147,158$ & 90 \\
\hline 6 & 13.58 & silanamine & $73,147,174$ & 83 \\
\hline 8 & 13.98 & 3,7-dioxa-2,8-disilanonane & $73,117,147,205$ & 90 \\
\hline 9 & 14.65 & glycine & $73,147,174,248$ & 83 \\
\hline 10 & 15.50 & propanoic acid & $73,147,189,292$ & 99 \\
\hline 11 & 19.52 & butanedioic acid & $73,147,233,245$ & 99 \\
\hline 12 & 20.10 & L-proline & $73,147,156$ & 90 \\
\hline 13 & 20.25 & furosardonin $\mathrm{A}$ & $73,100,147,218,232$ & 99 \\
\hline 14 & 22.54 & $N$-trimethylsylygrutam & $73,128,147,218,246$ & 90 \\
\hline 15 & 24.83 & xylitol & $73,147,205,217,307$ & 91 \\
\hline 16 & 25.85 & L-glutamic acid & $73,147,156,245$ & 96 \\
\hline 17 & 27.98 & D-galactose & $73,147,191,204,217$ & 95 \\
\hline 18 & 28.30 & fructose oxime & $73,103,147,217,307$ & 91 \\
\hline 19 & 28.68 & D-mannitol & $73,147,205,217,319$ & 87 \\
\hline 20 & 29.06 & D-sorbit & $73,147,205,319$ & 91 \\
\hline 21 & 29.41 & galactose benzyloxime & $73,147,205,217,319$ & 90 \\
\hline 22 & 29.42 & D-gluctiol & $73,147,205,217,319$ & 91 \\
\hline 23 & 30.43 & fructose & $73,103,217,307$ & 83 \\
\hline 24 & 32.31 & 1-naphthalenepentanoic acid & $47,73,217,305,318$ & 91 \\
\hline 25 & 34.38 & octadecanoic acid & $73,117,204,341$ & 99 \\
\hline 26 & 45.95 & melibiose & 73, 191, 204, 217 & 86 \\
\hline 27 & 46.40 & lyxopyranose & $73,141,191,204,217$ & 86 \\
\hline 28 & 48.28 & maltose & $73,204,217$ & 90 \\
\hline 29 & 52.11 & sucrose & $73,117,147,191$ & 81 \\
\hline
\end{tabular}

${ }^{a}$ full score at 100 . 


\subsection{Determining the Origin of G. elata and R. glutinosa Using Unsupervised Statistical Analysis}

The most popular unsupervised statistical method, PCA, was applied to the metabolomics data obtained from the four analytical methods (Figure 1). All the data tables were pre-processed with centering and scaling by unit variance, which corrected the variables by calculating the base weight of each variable as 1 standard deviation. $\mathrm{R}^{2} \mathrm{X}$ (cum) and $\mathrm{Q}^{2}$ (cum) values as the validation parameters of statistical models were displayed in Table 4.

Figure 1. PCA score plots of G. elata (a, FT-NIR; b, ${ }^{1} \mathrm{H}-\mathrm{NMR} ; \mathbf{c}$, GC-MS; d, LC-MS) and R. glutinosa (e, FT-NIR; f, ${ }^{1} \mathrm{H}-\mathrm{NMR}$; g, GC-MS; h, LC-MS). Red triangles for Chinese samples; black diamonds for Korean samples.
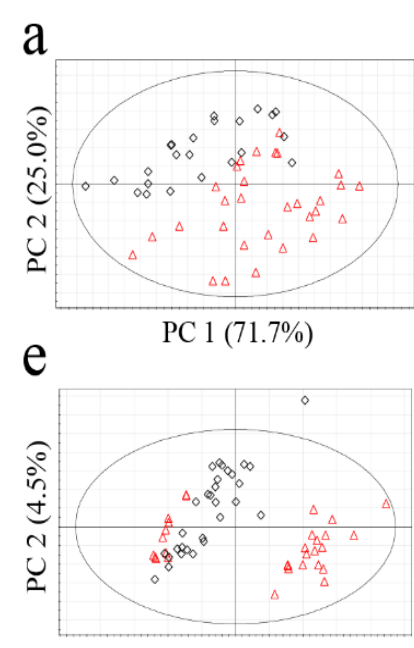

PC $1(95.0 \%)$ $\mathrm{b}$

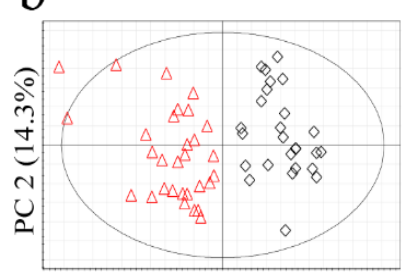

f

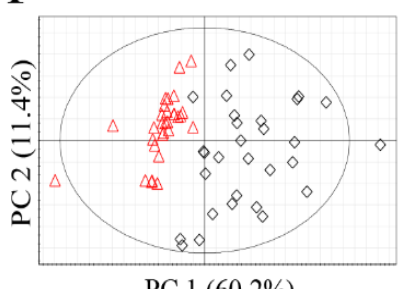

PC $1(60.2 \%)$
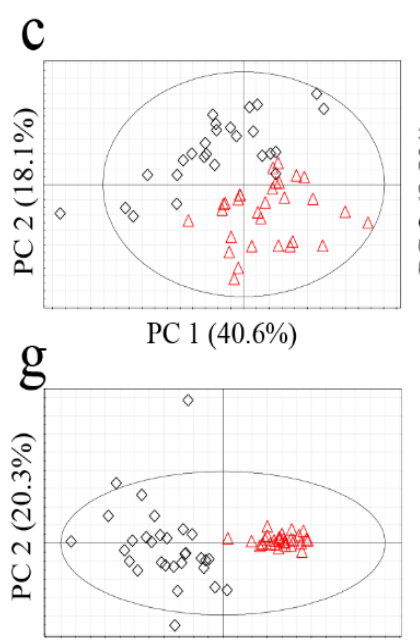

PC $1(35.1 \%)$
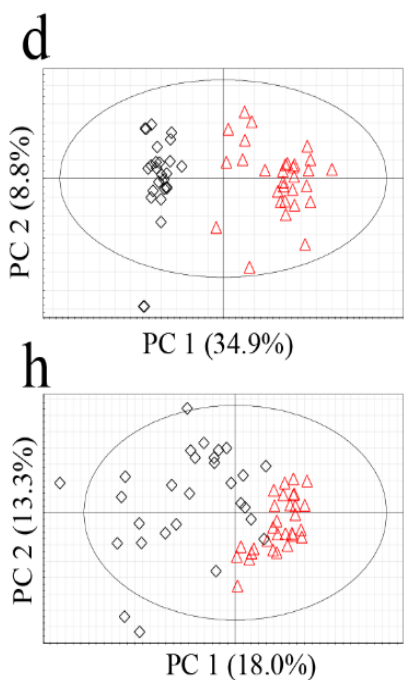

Table 4. Validation of the PCA models.

\begin{tabular}{cccc}
\hline Plants & Approaches & $\mathbf{R}^{\mathbf{2}} \mathbf{X}$ (cum) & $\mathbf{Q}^{\mathbf{2}}$ (cum) \\
\hline \multirow{4}{*}{ R. glutinosa } & FT-NIR & 0.951 & 0.948 \\
& ${ }^{1}$ H-NMR & 0.960 & 0.878 \\
& GC-MS & 0.869 & 0.734 \\
& LC-MS & 0.661 & 0.380 \\
\hline \multirow{5}{*}{ G. elata } & FT-NIR & 0.999 & 0.998 \\
& ${ }^{1}$ H-NMR & 0.887 & 0.812 \\
& GC-MS & 0.795 & 0.697 \\
& LC-MS & 0.695 & 0.469 \\
\hline
\end{tabular}

No clear classification of the origin (Korea or China) could be found from FT-NIR data (Figure 1a,e). Classification abilities of the statistical models were evaluated as a misclassification rate. The misclassification rate was calculated from the PC1 column plots (Figure 2), given that PC1 of all the models was the key component sufficient for the origin discrimination in the two plants as in Figure 1. The resulting misclassification rates for $G$. elata were $27 \%(8 / 30)$ and $30 \%(7 / 23)$ for Chinese and Korean samples, respectively. The overall rate was 28\% (15/53) (Figure 2a). The misclassification rates for $R$. glutinosa were 37\% (11/30) for Chinese and 17\% (5/30) for Korean samples, and the overall rate was 27\% (16/60) (Figure 2e). Though FT-NIR has been reported to be 
useful for discriminating the quality and geographical origin of green tea [24,25], its high misclassification rates in our study suggest that analysis using FT-NIR and PCA was not useful for discriminating the origin of the two medicinal plants tested, despite its simplicity and speed.

Figure 2. PCA column plots and misclassification rates of G. elata (a, FT-NIR;

b, ${ }^{1} \mathrm{H}-\mathrm{NMR}$; c, GC-MS; d, LC-MS) and R. glutinosa (e, FT-NIR; f, ${ }^{1} \mathrm{H}-\mathrm{NMR}$; g, GC-MS;

h, LC-MS). Red for Chinese samples; black for Korean samples.

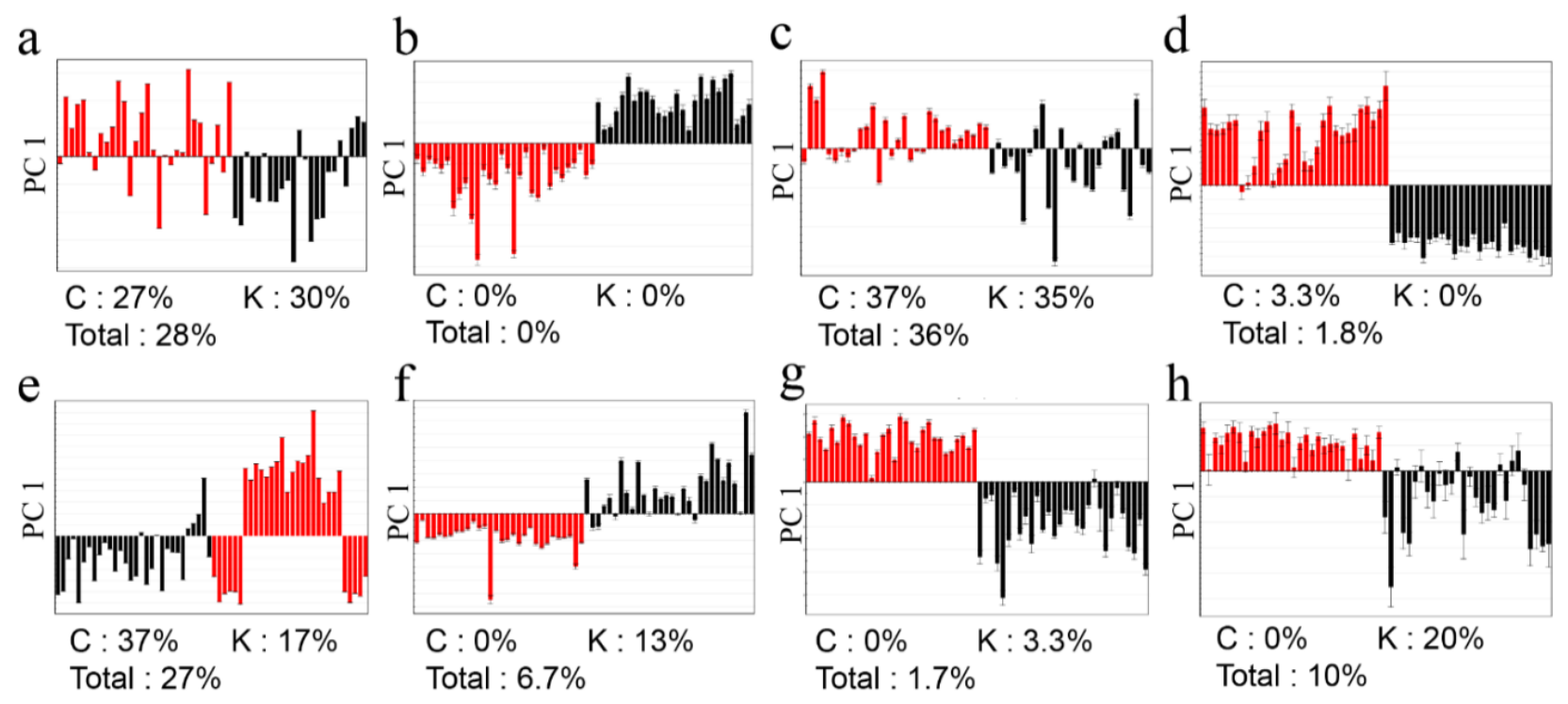

${ }^{1} \mathrm{H}-\mathrm{NMR}$ had much lower misclassification rates for both plants than FT-NIR (Figure 2b,f). For $G$. elata, no misclassifications were found, while two misclassifications were found for Korean R. glutinosa $(13 \% ; 4 / 30)$, making the overall rate $6.7 \%(4 / 60)$. These results imply that metabolites present at concentrations high enough to be detected by a relatively insensitive technique, such as ${ }^{1} \mathrm{H}-\mathrm{NMR}$, can be used to authenticate their origins.

In the GC-MS analysis (Figure 2c,g), the misclassification rates were inconsistent within the two species. That is, the misclassification rates were quite high (China, 37\%, 11/30; Korea, 35\%, 9/26) for G. elata, while there was only one misclassification for R. glutinosa (China, 0.0\%, 0/30; Korea, 3.3\%, $1 / 30)$. The overall misclassification rates from the LC-MS analysis were 1.8\% (1/56) for G. elata and $10 \%(6 / 60)$ for R. glutinosa (Figure 2d,h).

The distance in each discriminant model was not considered when calculating the misclassification rates above because of the inherent property of PCA. Samples of which relative distance to the maximum distance within a group was below 15\% were correctly classified. Therefore, these "ambiguous" samples contributed to the low misclassification rate, despite their short distances in the discriminant model (Figure 3). For example, Chinese sample No. 12 was categorized as Chinese in the plot, but was quite close to the Korean samples, and, therefore, could introduce some uncertainty. As a result, the supervised method described below was used to further evaluate the discriminative ability of the four methods. 
Figure 3. PC1 column plots of $R$. glutinosa analyzed by GC-MS (enlarged version of Figure 2g). Ambiguous samples and misclassified samples are indicated.

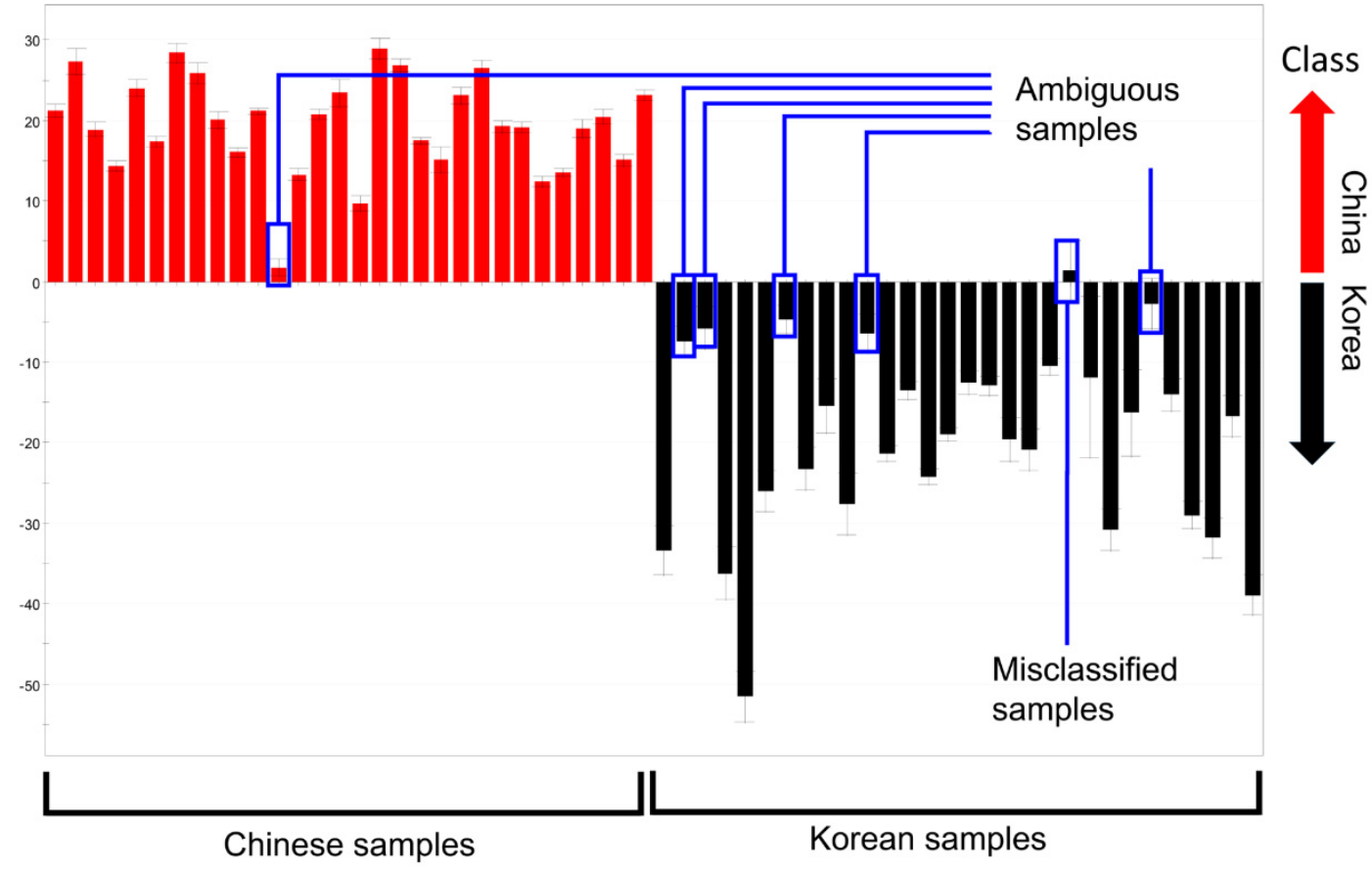

\subsection{Determination of the Origin of G. elata and R. glutinosa Using Supervised Statistical Analysis}

The metabolomics data were subjected to a supervised statistical analysis method, OPLS-DA, to measure the classifications of each model (Figure 4). All of the models exhibited high-quality parameters as summarized in Table 5, with overall values of $R^{2} Y$ (cum) and $Q^{2}$ (cum) close to 1.0. $p$-value from cross validated-analysis of variation (CV-ANOVA) as a way of verification of model validity indicated that the models were statistically significant (Table 5) [26].

Figure 4. OPLS-DA score plots of G. elata (FT-NIR; a, ${ }^{1} \mathrm{H}-\mathrm{NMR} ; \mathbf{b}, \mathrm{GC}-\mathrm{MS} ; \mathbf{c}, \mathrm{LC}-\mathrm{MS}$; d) and R. glutinosa (FT-NIR; e, ${ }^{1} \mathrm{H}-\mathrm{NMR}$; f, GC-MS; g, LC-MS; h). Red triangles for Chinese samples; black diamonds for Korean samples.

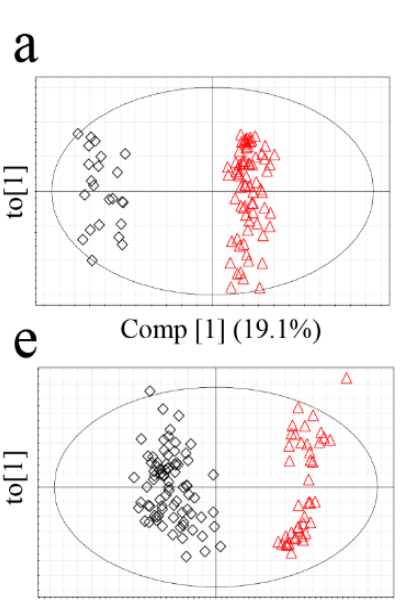

Comp [1] $(18.0 \%)$ b
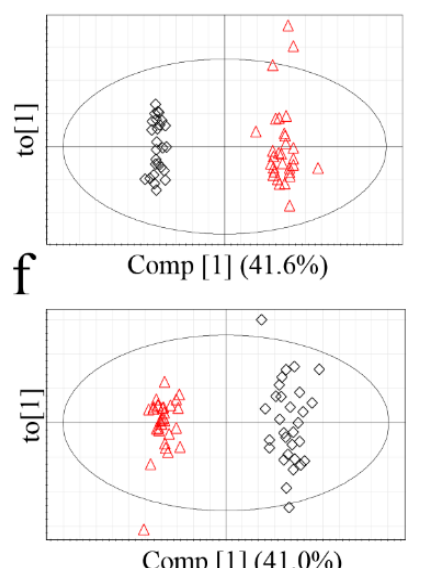

Comp [1] (41.0\%) c
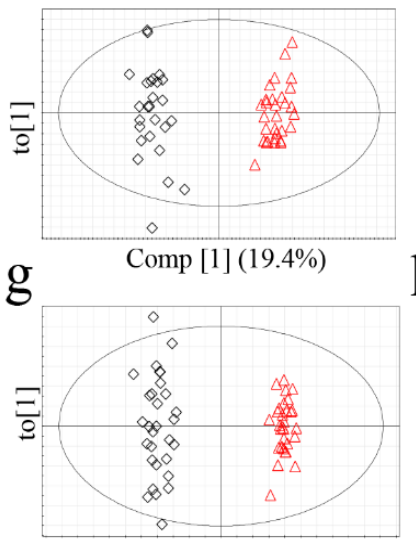

Comp [1] (26.9\%) d
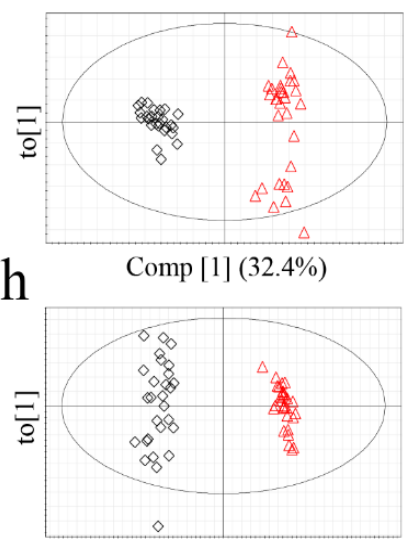

Comp [1] (15.1\%) 
Table 5. Validation of the OPLS-DA models and CV-ANOVA values of each model to manifest the model's regression.

\begin{tabular}{ccccc}
\hline Plants & Approaches & $\mathbf{R}^{2} \mathbf{Y}$ (cum) & $\mathbf{Q}^{\mathbf{2}}$ (cum) & $\boldsymbol{p}$-value of CV-ANOVA \\
\hline \multirow{3}{*}{ R. glutinosa } & FT-NIR & 0.933 & 0.937 & $7.06 \times 10^{-11}$ \\
\cline { 2 - 5 } & ${ }^{1} \mathrm{H}-\mathrm{NMR}$ & 0.969 & 0.937 & $7.98 \times 10^{-28}$ \\
\cline { 2 - 5 } & GC-MS & 0.971 & 0.904 & $3.33 \times 10^{-23}$ \\
\cline { 2 - 5 } & LC-MS & 0.967 & 0.952 & $2.74 \times 10^{-34}$ \\
\hline \multirow{3}{*}{ G. elata } & FT-NIR & 0.966 & 0.944 & 0 \\
\cline { 2 - 5 } & ${ }^{1} \mathrm{H}-\mathrm{NMR}$ & 0.982 & 0.973 & $1.13 \times 10^{-36}$ \\
\cline { 2 - 5 } & GC-MS & 0.969 & 0.924 & $1.11 \times 10^{-23}$ \\
\cline { 2 - 5 } & LC-MS & 0.970 & 0.951 & $1.25 \times 10^{-32}$ \\
\hline
\end{tabular}

Based on $\mathrm{Q}^{2}$ (cum) value of the OPLS-DA models, which is indicative of classification ability of model [27], applicability of each model for origin discrimination was evaluated. The most suitable analytical techniques for discriminating between the origin of $G$. elata and R. glutinosa were ${ }^{1} \mathrm{H}-\mathrm{NMR}$ and LC-MS, respectively. In the analysis of G. elata, ${ }^{1} \mathrm{H}-\mathrm{NMR}$ had the highest discriminative ability followed by LC-MS. This result is consistent with the misclassification rates calculated from PCA, which were lowest in ${ }^{1} \mathrm{H}-\mathrm{NMR}(0.0 \%)$ followed by LC-MS $(1.8 \%)$. These observations indicate that ${ }^{1} \mathrm{H}-\mathrm{NMR}$ and LC-MS-based approaches were appropriate for discrimination. On the other hand, for R. glutinosa, the $\mathrm{Q}^{2}$ value of the GC-MS analysis was lower than those of LC-MS and ${ }^{1} \mathrm{H}-\mathrm{NMR}$, and GC-MS had the lowest misclassification rate. Compared to the other techniques, GC-MS required much longer and more complicated sample preparation, including the derivatization steps, which could have lowered the reproducibility and possibly $\mathrm{Q}^{2}$. It is also conceivable that the unsupervised PCA performed better in analyzing the GC-MS data than the supervised OPLS-DA that is presumed to overfit the data by using class information in dimension reduction. The FT-NIR method, which had the shortest and most convenient sample preparation and analysis, was acceptable for determining the origin of G. elata and R. glutinosa based on OPLS-DA, though it was somewhat disqualified by PCA.

Upon integrating the results from the two statistical analyses, the best discrimination method differed for each plant species, which suggests that metabolomics approaches should be investigated on a case-by-case basis. Nonetheless, ${ }^{1} \mathrm{H}-\mathrm{NMR}$ and LC-MS were preferable for discriminating the origin of the two plants over the others.

\section{Experimental Section}

\subsection{Plant Materials}

The plants were collected from a number of major cultivation regions in Korea and China. A total of 26 G. elata samples were obtained from five cities in Korea (Chuncheon, Gimcheon, Muju, Sangju, and Asan), while 30 samples were obtained from Guangxi, Chongquing, Henan, Hunan, Shanxi, Anhui, Zhejiang, Hubei, and Guizhou in China. Thirty $R$. glutinosa samples were obtained from Andong, Jecheon, Geumsan, Seocheon, Jeongeup, and Hwasun in Korea, and 30 samples were obtained from Shanxi, Henan, and Hebei in China (Table 6). In each country, samples were collected from various farms in many cities that are relatively far from one another to represent a wide diversity 
of cultivation environments. The plant samples were dried for 3 days in an oven at $50{ }^{\circ} \mathrm{C}$ and ground with an electric mill (DA700, Daesung artlon, Seoul, Korea). Ground samples were sieved and particles between 125 and $300 \mu \mathrm{m}$ were used for analysis to ensure consistent extraction efficacy among the samples.

Table 6. Sample collected regions of two herbal medicines in Korea and China.

\begin{tabular}{|c|c|c|c|c|c|c|c|}
\hline \multicolumn{4}{|c|}{ G. elata } & \multicolumn{4}{|c|}{ R. glutinosa } \\
\hline Korea & $\begin{array}{c}\text { Sample } \\
\text { No. }\end{array}$ & China & $\begin{array}{c}\text { Sample } \\
\text { No. }\end{array}$ & Korea & $\begin{array}{c}\text { Sample } \\
\text { No. }\end{array}$ & China & $\begin{array}{c}\text { Sample } \\
\text { No. }\end{array}$ \\
\hline Chuncheon & 4 & Guangxi & 2 & Andong & 5 & Shanxi & 10 \\
\hline Gimcheon & 9 & Chongquing & 1 & Jecheon & 5 & Henan & 10 \\
\hline Muju & 3 & Henan & 3 & Geumsan & 5 & Hebei & 10 \\
\hline Sangju & 1 & Hunan & 3 & Seocheon & 5 & & \\
\hline \multirow[t]{5}{*}{ Asan } & 9 & Shanxi & 4 & Jeongeup & 5 & & \\
\hline & & Anhui & 3 & Hwasun & 5 & & \\
\hline & & Zhejiang & 3 & & & & \\
\hline & & Hubei & 10 & & & & \\
\hline & & Guizhou & 1 & & & & \\
\hline
\end{tabular}

\subsection{Near Infrared Spectroscopy}

Ground samples weighing $800 \mathrm{mg}$ were spread at bottom of an aluminum sample tube, and the spectrum of each sample was measured three times. For sampling, integrating sphere was employed to minimize heterogeneity and reduce deviation of baseline within data of each sample, given that it uses the diffuse reflectance. Samples were scanned from 12,000 to $4000 \mathrm{~cm}^{-1}$ with resolution of $8 \mathrm{~cm}^{-1}$ and each spectrum was obtained by averaging 32 scans using Fourier transform- near-infrared spectrometer (FT-NIR; MPA, Bruker optics, Rheinstetten, Germany). After data acquisition, data were preprocessed using multiple scattering correction (MSC).

\subsection{Nuclear Magnetic Resonance Spectroscopy}

The extraction solvent comprised 50\% methanol- $\mathrm{d}_{4}$ (Euriso-Top, Saint-Aubin, France) in deuterium oxide (Euriso-Top) with phosphate buffer at $\mathrm{pH} 6.0$ (sodium phosphate monobasic and dibasic, Sigma-Aldrich, Madrid, Spain). 3-(Trimethylsilyl)propionic-2,2,3,3,-d 4 acid (TMSP, Sigma-Aldrich) was added as an internal standard of chemical shift and to normalize intensity. One hundred mg of sample in $1.5 \mathrm{~mL}$ of extraction solvent was sonicated for $15 \mathrm{~min}$ at $25{ }^{\circ} \mathrm{C}$. The extracts were centrifuged at 13,000 $\mathrm{g}$ for $10 \mathrm{~min}$ and filtered using a $0.5-\mu \mathrm{m}$ syringe filter (Toyo Roshi Kaisha, Tokyo, Japan). The upper phase $(600 \mu \mathrm{L})$ was transferred to an NMR tube and analyzed with a JEOL ECA 500 spectrometer, equipped with a TH5 probe (JEOL, Tokyo, Japan). The parameters were as follows: $5.7 \mu \mathrm{s}\left(45^{\circ}\right)$ pulse, $9384.0 \mathrm{~Hz}$ spectral width, 8 scans, and $5 \mathrm{~s}$ relaxation delay with 64 transients collected in 32,000 data points. The residual water spectrum was pre-saturated within the relaxation delay $(4.79 \mathrm{ppm})$. 


\subsection{Liquid Chromatography-Mass Spectrometry}

One hundred $\mathrm{mg}$ of sample was sonicated in $1 \mathrm{~mL}$ of methanol (J.T. Baker, NJ, USA) for $30 \mathrm{~min}$ at $25{ }^{\circ} \mathrm{C}$. The extracts were centrifuged at $13,000 \mathrm{~g}$ for $10 \mathrm{~min}$ and filtered with a $0.2 \mu \mathrm{m}$ syringe filter (Toyo Roshi Kaisha, Otawa, Tokyo, Japan). Ultra high performance liquid chromatography (ACQUITY UPLC module, Waters Corporation, Milford, MA, USA) equipped with micro-TOF QII (Bruker Daltonik GmbH, Bremen, Germany) was used to separate and detect metabolites. Metabolites were separated on an ACQUITY UPLC BEH C18 column $(2.1 \times 100 \mathrm{~mm}, 1.7 \mu \mathrm{m}$, Waters Corporation) at a flow rate of $0.2 \mathrm{~mL} / \mathrm{min}$. Elution was performed using $0.1 \%$ formic acid (Sigma-Aldrich) in water (J.T. Baker) (solvent A) and $0.1 \%$ formic acid in acetonitrile (J.T. Baker) (solvent B) at the following gradient: $0 \% \mathrm{~B}$ at $0 \mathrm{~min}, 30 \% \mathrm{~B}$ at $5 \mathrm{~min}, 70 \% \mathrm{~B}$ at $15 \mathrm{~min}, 80 \% \mathrm{~B}$ at $25 \mathrm{~min}, 100 \% \mathrm{~B}$ at $27 \mathrm{~min}$, and held for $13 \mathrm{~min}$. The column was pre-equilibrated with $100 \% \mathrm{~A}$ for $10 \mathrm{~min}$. For ion detection, a positive mode was employed for both $R$. glutinosa and G. elata based on literatures reporting that the numbers of detected metabolites in the tested plants were similar in the positive and negative mode [28,29]. Electrospray ionization (ESI) was used at the following parameters: end plate offset, $500 \mathrm{eV}$; capillary voltage, $4500 \mathrm{eV}$; nebulizer, 1.2 bar; dry gas, $8.0 \mathrm{~mL} / \mathrm{min}$; capillary temperature, $200^{\circ} \mathrm{C}$; mass range, $49-1000 \mathrm{~m} / \mathrm{z}$.

\subsection{Gas Chromatography-Mass Spectrometry}

One $\mathrm{mL}$ of methanol was used to extract $10 \mathrm{mg}$ of sample, which was sonicated for $30 \mathrm{~min}$ at $25{ }^{\circ} \mathrm{C}$ and filtered through a $0.5 \mu \mathrm{m}$ syringe filter. Four hundred $\mu \mathrm{L}$ of filtered extract was purged under $\mathrm{N}_{2}$. After lyophilization, the extract was reconstituted in $50 \mu \mathrm{L}$ of methoxyamine hydrochloride in pyridine (20 mg/mL, Sigma-Aldrich) and heated at $30{ }^{\circ} \mathrm{C}$ for $90 \mathrm{~min}$ for methoxymation of the carbonyl group. Then, $100 \mu \mathrm{L}$ of N,O-bis(trimethylsilyl)trifluoroacetamide (BSTFA) with 1\% trimethylchlorosilane (TMCS) (Sigma-Aldrich) was added and heated at $37{ }^{\circ} \mathrm{C}$ for $30 \mathrm{~min}$. GC-MS (6890A connected to 5973 MSD, Agilent technologies, DE, USA) with a DB-5 column $(30 \mathrm{~m} \times 0.25 \mathrm{~mm}, 250 \mu \mathrm{m}$ thickness, Agilent technologies) was used for analysis. The injection volume was $1 \mu \mathrm{L}$ with a 1:30 split and an injection temperature of $280^{\circ} \mathrm{C}$. The carrier gas was helium at a flow rate of $0.5 \mathrm{~mL} / \mathrm{min}$. The oven temperature was as follows: initially $80{ }^{\circ} \mathrm{C}$ for $2 \mathrm{~min}$, increased to $150{ }^{\circ} \mathrm{C}$ at $5{ }^{\circ} \mathrm{C} / \mathrm{min}$, held at $150{ }^{\circ} \mathrm{C}$ for $2 \mathrm{~min}$, increased to $300{ }^{\circ} \mathrm{C}$ at $5{ }^{\circ} \mathrm{C} / \mathrm{min}$, and held at $300{ }^{\circ} \mathrm{C}$ for $20 \mathrm{~min}$. Electron impact with $70 \mathrm{eV}$ was used to ionize samples and acquire data under scan mode at 50-500 m/z.

\subsection{Data Analysis}

Peaks from the GC-MS analysis were assigned using the NIST mass spectral library [30]. ${ }^{1} \mathrm{H}-\mathrm{NMR}$ peak assignment was performed using the BMRB database (Biological Magnetic Resonance Bank) [31] and data from the literature [32-34].

Datasets acquired from the FT-NIR analysis were exported and binned using Unscrambler software [35]. ${ }^{1} \mathrm{H}-\mathrm{NMR}$ data were referenced to TMSP to calibrate chemical shifts (at $0.00 \mathrm{ppm}$ ). After calibration, the datasets were exported to ASCII format, after which $0.2-10 \mathrm{ppm}$ tables were reduced to $0.04 \mathrm{ppm}$ buckets. Four bins $(3.29-3.37 \mathrm{ppm})$ corresponding to solvent residual peaks were removed, leaving 239 bins. ${ }^{1} \mathrm{H}-\mathrm{NMR}$ data pre-processing including peak alignment, normalization, and 
binning was performed with MNova (Mestrelab research, Santiago, Spain). In the LC-MS analysis, the extracted ion chromatogram of a specific $\mathrm{m} / \mathrm{z}$ at a specific retention time, which corresponds to one variable in the statistical analysis, was aligned using MZmine [36] with RANSAC (RANdom SAmple Consensus) algorithm, after which the tables of aligned data were used in multivariate statistical analysis including principal component analysis (PCA) and orthogonal projection to latent structuresdiscriminant analysis (OPLS-DA) with SIMCA-P ${ }^{+}$[37].

\section{Conclusions}

Four metabolomics approaches were applied to determine the regional origin of $G$. elata and R. glutinosa. Their classification abilities were evaluated based on the misclassification rates and $\mathrm{Q}^{2}$ values acquired from PCA and OPLS-DA, respectively. The best discrimination method differed for each plant species, i.e., ${ }^{1} \mathrm{H}-\mathrm{NMR}$ and LC-MS were found as the best techniques for G. elata and R. glutinosa, respectively. Reasoned by integrating all the results from the two statistical methods, ${ }^{1} \mathrm{H}-\mathrm{NMR}$ was generally the most prominent technique for discriminating the origins of G. elata and $R$. glutinosa. However, our study suggests that preliminary screening for the most suitable analytical tool and statistical method is essential to ensure the dependability of metabolomics-based discrimination of regional origins.

\section{Supplementary Materials}

Supplementary materials can be accessed at: http://www.mdpi.com/1420-3049/19/5/6294/s1.

\section{Acknowledgments}

This work was supported by the following grants: the Next-Generation BioGreen 21 Program (No. PJ008202) from the Rural Development Administration, Republic of Korea, the Basic Science Research Program (NRF-2013R1A1A1A05005753) from the National Research Foundation of Korea (NRF), and the Bio-Synergy Research Project (NRF-2012M3A9C4048796) from the NRF funded by the Ministry of Science, ICT and Future Planning. This work was also partially supported by the Ministry of Health and Welfare, Republic of Korea (2010) and Dongduk Women's University's sabbatical leave.

\section{Author Contributions}

H.J. Cho, J.H. Park, Y.B. Seo, S.W. Kwon, and J. Lee designed research. D.-K. Lee, D.K. Lim, J.A. Um, C.J. Lim, Y.A. Yoon, and Y. Ryu performed experiments. D.-K. Lee, H.J. Kim, K. Kim, J. Lim, S.W. Kwon, and J. Lee analyzed data. D.-K. Lee, J. Y. Hong, S.W. Kwon, and J. Lee wrote manuscript.

\section{Conflicts of Interest}

The authors declare no conflict of interest. 


\section{References}

1. Park, H.J.; Kim, D.H.; Park, S.J.; Kim, J.M.; Ryu, J.H. Ginseng in traditional herbal prescriptions. J. Ginseng Res. 2012, 36, 225-241.

2. Joos, S.; Glassen, K.; Musselmann, B. Herbal Medicine in Primary Healthcare in Germany: The Patient's Perspective. Evid. Based Complement. Alternat. Med. 2012, 2012, doi:10.1155/2012/294638.

3. Guerra, M.C.; Speroni, E.; Broccoli, M.; Cangini, M.; Pasini, P.; Minghetti, A.; Crespi-Perellino, N.; Mirasoli, M.; Cantelli-Forti, G.; Paolini, M. Comparison between Chinese medical herb Pueraria lobata crude extract and its main isoflavone puerarin: Antioxidant properties and effects on rat liver CYP-catalysed drug metabolism. Life Sci. 2000, 67, 2997-3006.

4. Kim, H.J.; Kim, P.; Shin, C.Y. A comprehensive review of the therapeutic and pharmacological effects of ginseng and ginsenosides in central nervous system. J. Ginseng Res. 2013, 37, 8-29.

5. Lydon, J.; Duke, S.O. Pesticide effects on secondary metabolism of higher plants. Pest. Sci. 1989, $25,361-373$.

6. Ramakrishna, A.; Ravishankar, G.A. Influence of abiotic stress signals on secondary metabolites in plants. Plant Signal. Behav. 2011, 6, 1720-1731.

7. Palumbo, M.; Putz, F.; Talcott, S. Nitrogen fertilizer and gender effects on the secondary metabolism of yaupon, a caffeine-containing North American holly. Oecologia 2007, 151, 1-9.

8. Stenseth, N.C.; Mysterud, A.; Ottersen, G.; Hurrell, J.W.; Chan, K.-S.; Lima, M. Ecological Effects of Climate Fluctuations. Science 2002, 297, 1292-1296.

9. Wang, J.-B.; Zeng, L.-N.; Zang, Q.-C.; Gong, Q.-F.; Li, B.-C.; Zhang, X.-R.; Chu, X.-H.; Zhang, P.; Zhao, Y.-L.; Xiao, X.-H. Colorimetric Grading Scale Can Promote the Standardization of Experiential and Sensory Evaluation in Quality Control of Traditional Chinese Medicines. PLoS One 2012, 7, e48887.

10. Vergouw, C.G.; Botros, L.L.; Roos, P.; Lens, J.W.; Schats, R.; Hompes, P.G.A.; Burns, D.H.; Lambalk, C.B. Metabolomic profiling by near-infrared spectroscopy as a tool to assess embryo viability: A novel, non-invasive method for embryo selection. Hum. Reprod. 2008, 23, 1499-1504.

11. López-Rituerto, E.; Savorani, F.; Avenoza, A.; Busto, J.H.; Peregrina, J.M.; Engelsen, S.B. Investigations of La Rioja Terroir for Wine Production Using ${ }^{1} \mathrm{H}$ NMR Metabolomics. J. Agric. Food Chem. 2012, 60, 3452-3461.

12. Luo, F.; Lu, R.; Zhou, H.; Hu, F.; Bao, G.; Huang, B.; Li, Z. Metabolic Effect of an Exogenous Gene on Transgenic Beauveria bassiana Using Liquid Chromatography-Mass Spectrometry-Based Metabolomics. J. Agric. Food Chem. 2013, 61, 7008-7017.

13. Cevallos-Cevallos, J.M.; Futch, D.B.; Shilts, T.; Folimonova, S.Y.; Reyes-De-Corcuera, J.I. GC-MS metabolomic differentiation of selected citrus varieties with different sensitivity to citrus huanglongbing. Plant Physiol. Biochem. 2012, 53, 69-76.

14. Botros, L.; Sakkas, D.; Seli, E. Metabolomics and its application for non-invasive embryo assessment in IVF. Mol. Hum. Reprod. 2008, 14, 679-690.

15. Baek, S.H.; Bae, O.N.; Park, J.H. Recent methodology in ginseng analysis. J. Ginseng Res. 2012, 36, 119-134.

16. Moco, S.; Vervoort, J.; Bino, R.J.; de Vos, R.C.H.; Bino, R. Metabolomics technologies and metabolite identification. Trac-Trends Anal. Chem. 2007, 26, 855-866. 
17. Dunn, W.B.; Ellis, D.I. Metabolomics: Current analytical platforms and methodologies. Trac-Trends Anal. Chem. 2005, 24, 285-294.

18. Zhang, R.; Zhou, J.; Jia, Z.; Zhang, Y.; Gu, G. Hypoglycemic effect of Rehmannia glutinosa oligosaccharide in hyperglycemic and alloxan-induced diabetic rats and its mechanism. J. Ethnopharmacol. 2004, 90, 39-43.

19. Liang, A.; Xue, B.; Wang, J.; Hao, J.; Yang, H.; Yi, H. A study on hemostatic and immunological actions of fresh and dry Dihuang. Zhongguo Zhong Yao Za Zhi 1999, 24, 663-666, 702.

20. Hsieh, C.-L.; Tang, N.-Y.; Chiang, S.-Y.; Hsieh, C.-T.; Lin, J.-G. Anticonvulsive and free radical scavenging actions of two herbs, Uncaria rhynchophylla (Miq) jack and Gastrodia elata B1., in kainic acid-treated rats. Life Sci. 1999, 65, 2071-2082.

21. Ahn, E.-K.; Jeon, H.-J.; Lim, E.-J.; Jung, H.-J.; Park, E.-H. Anti-inflammatory and anti-angiogenic activities of Gastrodia elata Blume. J. Ethnopharmacol. 2007, 110, 476-482.

22. William Allwood, J.; Ellis, D.I.; Heald, J.K.; Goodacre, R.; Mur, L.A.J. Metabolomic approaches reveal that phosphatidic and phosphatidyl glycerol phospholipids are major discriminatory non-polar metabolites in responses by Brachypodium distachyon to challenge by Magnaporthe grisea. Plant J. 2006, 46, 351-368.

23. Lee, D.-K.; Yoon, M.H.; Kang, Y.P.; Yu, J.; Park, J.H.; Lee, J.; Kwon, S.W. Comparison of primary and secondary metabolites for suitability to discriminate the origins of Schisandra chinensis by GC/MS and LC/MS. Food Chem. 2013, 141, 3931-3937.

24. Ikeda, T.; Kanaya, S.; Yonetani, T.; Kobayashi, A.; Fukusaki, E. Prediction of Japanese Green Tea Ranking by Fourier Transform Near-Infrared Reflectance Spectroscopy. J. Agric. Food Chem. 2007, 55, 9908-9912.

25. Chen, Q.; Zhao, J.; Lin, H. Study on discrimination of Roast green tea (Camellia sinensis L.) according to geographical origin by FT-NIR spectroscopy and supervised pattern recognition. Spectroc. Acta Pt. A-Molec. Biomolec. Spectr. 2009, 72, 845-850.

26. Eriksson, L.; Trygg, J.; Wold, S. CV-ANOVA for significance testing of PLS and OPLS ${ }^{\circledR}$ models. J. Chemometr. 2008, 22, 594-600.

27. Westerhuis, J.A.; Hoefsloot, H.C.J.; Smit, S.; Vis, D.J.; Smilde, A.K.; Velzen, E.J.J.; Duijnhoven, J.P.M.; Dorsten, F.A. Assessment of PLSDA cross validation. Metabolomics 2008, 4, 81-89.

28. Wang, L.; Xiao, H.; Liang, X.; Wei, L. Identification of phenolics and nucleoside derivatives in Gastrodia elata by HPLC-UV-MS. J. Sep. Sci. 2007, 30, 1488-1495.

29. Su, S.; Cui, W.; Zhou, W.; Duan, J.A.; Shang, E.; Tang, Y. Chemical fingerprinting and quantitative constituent analysis of Siwu decoction categorized formulae by UPLC-QTOF/MS/MS and HPLC-DAD. Chin. Med. 2013, 8, 5.

30. National Institute of Standards and Technology, version 2008; NIST Mass spectrometry Data Center: Gaithersburg, MD, USA, 2008.

31. Biological Magnetic Resonance Bank. Available online: http://www.bmrb.wisc.edu/ (accessed on 1 October 2013).

32. Li, N.; Wang, K.J.; Chen, J.J.; Zhou, J. Phenolic compounds from the rhizomes of Gastrodia elata. J. Asian Nat. Prod. Res. 2007, 9, 373-377. 
33. Yang, X.-D.; Zhu, J.; Yang, R.; Liu, J.-P.; Li, L.; Zhang, H.-B. Phenolic constituents from the rhizomes of Gastrodia elata. Nat. Prod. Res. 2007, 21, 180-186.

34. Morota, T.; Sasaki, H.; Nishimura, H.; Sugama, K.; Chin, M.; Mitsuhashi, H. Two iridoid glycosides from Rehmannia glutinosa. Phytochemistry 1989, 28, 2149-2153.

35. Unscrambler, version 9.1; CAMO: Trondheim, Norway, 1999.

36. Mzmine, version 2.10; Available online: http://mzmine.sourceforge.net/ (accessed on 11 November 2013).

37. $S I M C A-P^{+}$, version 12.0; Umetrics: Umeå, Sweden, 2008.

Sample Availability: Samples of the $R$. glutinosa and G. elata plant materials are available from the authors.

(C) 2014 by the authors; licensee MDPI, Basel, Switzerland. This article is an open access article distributed under the terms and conditions of the Creative Commons Attribution license (http://creativecommons.org/licenses/by/3.0/). 